Cohabitation effect on the slowdown of the Neolithic expansion

This article has been downloaded from IOPscience. Please scroll down to see the full text article.

2011 EPL 9658002

(http://iopscience.iop.org/0295-5075/96/5/58002)

View the table of contents for this issue, or go to the journal homepage for more

Download details:

IP Address: 84.88.138.106

The article was downloaded on 03/04/2013 at 09:31

Please note that terms and conditions apply. 


\title{
Cohabitation effect on the slowdown of the Neolithic expansion
}

\author{
N. $\operatorname{IsERN}^{(\mathrm{a})}$ and J. ForT \\ Complex System Lab, Departament de Física, Universitat de Girona - 17071 Girona, Catalonia, Spain, EU
}

received 16 May 2011; accepted in final form 8 October 2011

published online 15 November 2011

PACS 87.23.Cc - Population dynamics and ecological pattern formation

PACS 89.20.-a - Interdisciplinary applications of physics

PACS 89.65.Ef - Social organizations; anthropology

\begin{abstract}
We introduce the effect of cohabitation between generations to a previous model on the slowdown of the Neolithic transition in Europe. This effect consists on the fact that human beings do not leave their children alone when they migrate, but on the contrary they cohabit until their children reach adulthood. We also use archaeological data to estimate the variation of the Mesolithic population density with distance, and use this information to predict the slowdown of the Neolithic front speed. The new equation leads to a substantial correction, up to $37 \%$, relative to previous results. The new model is able to provide a satisfactory explanation not only to the relative speed but also to the absolute speed of the Neolithic front obtained from archaeological data.
\end{abstract}

Copyright (c) EPLA, 2011

Introduction. - Reaction-diffusion models have been applied to model many biological and cross-disciplinary complex systems such as the Neolithic transition, viral infections or tumor growth (for recent reviews see $[1,2]$ ).

The change from hunter-gathering economics to farming (known as Neolithic transition) in Europe is widely regarded as an invasion of farmers from the Near East. This process has been analyzed in several studies using physical and mathematical models [3-6]. A recent paper [3] presented a model to explain the slowdown of the Neolithic transition in Europe as higher latitudes were reached. It is known that the density of Mesolithic (i.e., hunter-gatherer) populations was higher at northern regions [7], and the model in reference [3] includes the effect of encountering these pre-Neolithic populations both in the dispersion and the reaction (or population growth) processes.

Another cause that one could expect to have a noticeable effect on the slowdown of the Neolithic transition is the adaptation of agriculture to temperate climates. However, this effect was, in fact, minimal because, according to the archaeological findings by Coward et al. [8], when establishing new settlements the Neolithic populations cultivated the more adaptable crops and simply dropped the less productive ones.

Some authors have also studied the effect of the geography on the front dynamics. Ackland et al. [9] stud-

\footnotetext{
(a) E-mail: neus . isem@udg.edu
}

ied this effect in terms of land fertility and obtained a decrease on the front speed when encountering mountains (regions of low fertility). In their model they assumed that the diffusion coefficient $D$ and the growth rate $a$ decreased at the mountains. However, we are not aware of any population data showing a decrease of $a$ with altitude and ethnographical data show that, in fact, $D$ increases in regions with low population densities [10,11], such as mountainous areas. On the other hand, Fort, Pujol and Vander Linden [12] have recently modeled the spread of the Neolithic in Europe taking into account the mountains as barriers, finding that the effect on the front speed is negligible at continental scale.

A simple and practical way of describing the evolution of the population density of farmers $N(x, y, t)$ is by assuming that its variation after a generation time $T$ is the sum of the variations due to dispersion and population growth. In such a model, the Neolithic population density at position $(x, y)$ and time $t+T$ would be

$$
\begin{aligned}
N(x, y, t+T)= & \iint N\left(x-\Delta_{x}, y-\Delta_{y}, t\right) \\
& \times \phi(x, y ; \theta, \Delta) \mathrm{d} \Delta_{x} \mathrm{~d} \Delta_{y}+R[N(x, y, t)],
\end{aligned}
$$

where the dispersion kernel $\phi(x, y ; \theta, \Delta)$ gives the probability that an individual initially at $\left(x-\Delta_{x}, y-\Delta_{y}\right)$ jumps a distance $\Delta$ in the direction $\theta$ during a generation time $T$, therefore reaching position $(x, y)$, with $\Delta=\sqrt{\Delta_{x}^{2}+\Delta_{y}^{2}}$ 
and $\theta=\tan ^{-1}\left(\Delta_{y} / \Delta_{x}\right)$. In a recent model for the slowdown of the Neolithic [3], it was shown that if the jump distance $\Delta$ is proportional to the free space in the final location, then the dispersion kernel for the Neolithic population $N$ can be written as

$$
\phi(x, y ; \theta, \Delta)=\frac{1}{2 \pi}\left[1-\frac{\partial M / \partial y}{M_{\max }-M} \Delta \sin \theta\right] \psi(\Delta),
$$

where $M(y)$ is the Mesolithic population density (assumed independent of $x$ for simplicity), $M_{\max }$ is the Mesolithic saturation density (i.e., the maximum possible value of $M(y))^{1}$, and $\psi(\Delta)$ is a function dependent only on the jump distance $\Delta$, normalized such that $\int_{0}^{\infty} \Delta \psi(\Delta) \mathrm{d} \Delta=1$.

The last term in eq. (1) gives the variation in Neolithic population density due to population growth (reproduction minus deaths) during a generation time $T$. This can generally be expressed as a Taylor series

$$
R[N(x, y, t)]=T F+\frac{T^{2}}{2} \frac{\partial F}{\partial t}+\frac{T^{3}}{3 !} \frac{\partial^{2} F}{\partial t^{2}}+\ldots,
$$

where $F=\left.\frac{\partial N}{\partial t}\right|_{g}$ is called the growth function and the subindex $g$ stands for the growth (as opposed to dispersion) process.

The presence of indigenous populations has an effect also on the growth function $F$. This can be taken into account by noting that the free space available for Neolithic individuals is reduced by $M / M_{\max }$ in addition to the usual logistic saturation term $N / N_{\max }$. It has been shown [3] that $F$ in eq. (3) is then given by

$$
F=a N\left(1-\frac{N}{N_{\max }}-\frac{M}{M_{\max }}\right),
$$

where $N$ is the Neolithic population density, $N_{\max }$ the saturation density for the Neolithic population and $a$ is called the initial growth rate for the Neolithic population.

In reference [3], in order to model the slowdown of the Neolithic front speed, the kernel (2) and the growth function (4) were applied to the evolution equation (1), from which the following equation for the front speed was found

$$
c=\sqrt{4 D \tilde{a}}-2 D \frac{\partial M / \partial y}{M_{\max }-M},
$$

where we have defined $\tilde{a} \equiv a\left(1-M / M_{\max }\right)$ and $D \equiv\left\langle\Delta^{2}\right\rangle / 4 T$. However, even though eq. (1) is often used for population dynamics, it is not realistic to describe human populations. Indeed, eq. (1) describes a system in which, after a generation time $T$, new individuals (children) will appear at $(x, y)$ while the parent population has already moved to $\left(x+\Delta_{x}, y+\Delta_{y}\right)$. However, although this behavior may be true for other species (like fish), human populations migrate with their

\footnotetext{
${ }^{1}$ Alternatively, $M$ can be interpreted as the local Mesolithic carrying capacity and $M_{\max }$ as its maximum possible value. This alternative interpretation does not change any of the equations and results in the present paper.
}

children (because the latter cannot survive on their own until adulthood). Thus, it has been stressed $[1,13,14]$ that an evolution equation modeling this cohabitation between parents and children should better represent human population dynamics. For this reason, in fact population growth should be applied to the dispersed population rather than to the initial one, i.e., the new population (children) appear where parents have moved. Then eq. (1) is replaced by $[1,13,14]$

$$
\begin{aligned}
& N(x, y, t+T)= \\
& \quad \iint N\left(x-\Delta_{x}, y-\Delta_{y}, t\right) \phi(x, y ; \theta, \Delta) \mathrm{d} \Delta_{x} \mathrm{~d} \Delta_{y} \\
& \quad+R\left[\iint N\left(x-\Delta_{x}, y-\Delta_{y}, t\right) \phi(x, y ; \theta, \Delta) \mathrm{d} \Delta_{x} \mathrm{~d} \Delta_{y}\right] .
\end{aligned}
$$

Alternatively, instead of eq. (6) one could also write down a cohabitation model where the reaction takes place initially $^{2}$, but they would both lead to the same front speed [13]. Equation (6) has been applied before [13,14] but never using the non-isotropic kernel (2) and modified growth function (4).

In this paper we will find the front speed for eq. (6) using the kernel (2) and growth function (4). We will apply the results to the slowdown of the Neolithic expansion in Europe and compare them with those from eq. (5) as well as with archaeological data.

Cohabitation model. - In order to derive a reactiondiffusion equation from the cohabitation equation (6) with the kernel (2) and the growth function (4), we first Taylorexpand this equation up to first order in time. This yields

$$
\begin{aligned}
& N(x, y, t)+T \frac{\partial N}{\partial t}= \\
& \quad \iint N\left(x-\Delta_{x}, y-\Delta_{y}, t\right) \phi(x, y ; \theta, \Delta) \mathrm{d} \Delta_{x} \mathrm{~d} \Delta_{y} \\
& \quad+T F\left[\iint N\left(x-\Delta_{x}, y-\Delta_{y}, t\right) \phi(x, y ; \theta, \Delta) \mathrm{d} \Delta_{x} \mathrm{~d} \Delta_{y}\right]
\end{aligned}
$$

Now, since our aim is to find an expression for the front speed, we can apply that at the leading edge of the front the Neolithic population density is $N \ll N_{\max }$. Thus, at the front we can linearize the growth equation $F$, eq. (4), as follows:

$$
F \approx a N\left(1-\frac{M}{M_{\max }}\right), \text { when } N \ll N_{\max } .
$$

We now Taylor-expand eq. (7) up to second order in space using the dispersion kernel (2) and the linearized approximation for the growth function (8), and we find the following differential equation (which is valid at the

\footnotetext{
${ }^{2}$ Then the last term in eq. (6) would be $\iint R\left[N\left(x-\Delta_{x}, y-\right.\right.$ $\left.\left.\Delta_{y}, t\right)\right] \phi(x, y ; \theta, \Delta) \mathrm{d} \Delta_{x} \mathrm{~d} \Delta_{y}$.
} 
leading edge of the expanding front):

$$
\begin{aligned}
\frac{\partial N}{\partial t}= & \tilde{a} N+2 D(1+T \tilde{a}) \frac{\partial M / \partial y}{M_{\max }-M} \frac{\partial N}{\partial y} \\
& +D(1+T \tilde{a})\left(\frac{\partial^{2} N}{\partial x^{2}}+\frac{\partial^{2} N}{\partial y^{2}}\right)
\end{aligned}
$$

where again we have used $\tilde{a} \equiv a\left(1-M / M_{\max }\right)$ and $D \equiv$ $\left\langle\Delta^{2}\right\rangle / 4 T$.

To find the front speed we note that for $t \rightarrow \infty$ the spreading front can be considered as locally planar, thus for $x=0$ and $y \rightarrow \infty$ the local speed $c$ is parallel to the $y$ axis [1]. We therefore look for constant-shaped solutions to eq. (9) with the form $N=N_{0} \exp [-\lambda(y-c t)]$ as $(y-c t) \rightarrow$ $\infty$, with $c>0$ and $\lambda>0$. Since $\lambda$ has to be real, we find that the front speed $c$ satisfies

$$
c \geqslant \sqrt{4 D \tilde{a}(1+T \tilde{a})}-2 D(1+T \tilde{a}) \frac{\partial M / \partial y}{M_{\max }-M} .
$$

Equation (10) gives a lower bound for the front speed in our model. However, it is easy to apply variational analysis [15] to the differential equation (9) and derive an upper bound for the front speed, which is again given by the same expression as in eq. (10). Thus, the exact speed for the front speed is ${ }^{3}$

$$
c=\sqrt{4 D \tilde{a}(1+T \tilde{a})}-2 D(1+T \tilde{a}) \frac{\partial M / \partial y}{M_{\max }-M} .
$$

Application to the Neolithic transition. - Here we will apply eq. (11) to the Neolithic transition in Europe and compare the front speeds predicted by this cohabitation equation with those from the non-cohabitation equation (5) and also with archaeological data.

Archaeological data have been used to estimate Neolithic front speeds within a rectangular region about $1300 \mathrm{~km}$ long and $400 \mathrm{~km}$ wide comprised between the Balkans and the North Sea (see fig. 1 in ref. [3], which defines the region and the $y$-direction). We constructed a map of arrival times of the Neolithic in Europe by interpolating 765 early Neolithic dates published by Pinhasi [16] (fig. 1 in ref. [3]). The front speed was estimated by computing the areas within isochrones separated 250 years inside the region of study. Here we compare the absolute speeds calculated for this region to our new cohabitation model (11) and the previously known non-cohabitation model (5) (in contrast, ref. [3] only dealt with data for the relative speed $c / c_{\max }$, with $c_{\max }=2 \sqrt{a D}$, and the non-cohabitation model (5)).

In order to compare the predictions from the models with the archaeological speeds, we will take into account that the anthropological parameters appearing in the models have been estimated as $a=0.028 \mathrm{y}^{-1}$ [14] for the initial growth rate for farmer populations, $T=32 \mathrm{y}$ [17] for the generation time and $\left\langle\Delta^{2}\right\rangle=1531 \mathrm{~km}^{2}$ [4] for the mean-squared displacement per generation.

\footnotetext{
${ }^{3}$ If there is no Mesolithic population $(\partial M / \partial y=0, M=0$ and $\tilde{a}=a)$ eq. (11) becomes $c=\sqrt{4 D a(1+T a)}$. If we take into account that $R_{0}=\exp (a T) \simeq 1+a T$ (see note [26] in ref. [13]), this agrees with eq. (23) in ref. [13] up to first order in time, as it should.
}

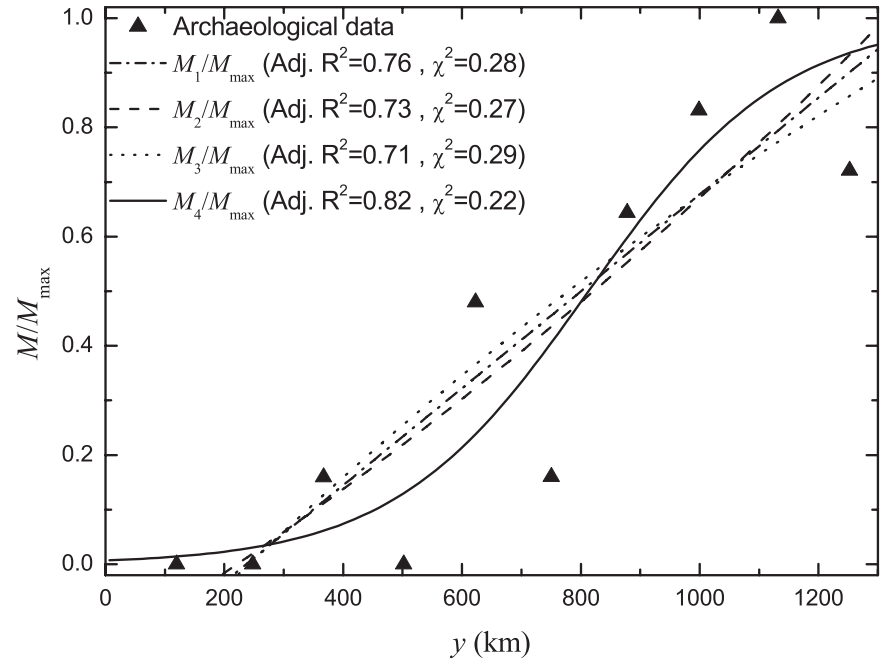

Fig. 1: Symbols: increase of the relative density of Mesolithic sites at Northern Europe calculated from archaeological data [20]. Lines: best fits for four possible models (eq. (12)) describing the variation of Mesolithic density.

The actual distribution of Mesolithic population in space, $M(y)$, is unknown due to the methodological difficulties in estimating population densities from archaeological data [18] and the lack of comprehensive publications on the Mesolithic. Fortunately, however, the speeds (5) and (11) depend only on the relative density $M(y) / M_{\max }$ (not on $M(y)$ and $M_{\max }$ separately), and archaeologists have stressed that it is reasonable to assume that the density of archaeological remains is proportional to that of the population [19]. Therefore, we can use relative densities of archaeological sites to estimate the function $M(y) / M_{\max }$, as necessary to apply eqs. (5) and (11). For this purpose, we have used the records on the Mesolithic period from the Radiocarbon Paleolithic Database Europe [20,21]. The density of sites has been computed at positions (values of $y$ along the rectangle in fig. 1 in ref. [3]) separated $130 \mathrm{~km}$, by counting the number of Mesolithic sites within an area of $260 \times 600 \mathrm{~km}$ surrounding each point ${ }^{4}$. Figure 1 shows the relative densities of sites obtained in our analysis (triangles), where $M_{\max }$ is the maximum measured density (the Mesolithic database is available at [21]). We have fitted these results to four possible models for the variation of $M$ in space,

$$
\begin{aligned}
& M_{1} / M_{\max }=A_{1} y+B_{1}, \\
& M_{2} / M_{\max }=A_{2}+B_{2} \exp \left(y / \tau_{2}\right), \\
& M_{3} / M_{\max }=A_{3}-B_{3} \exp \left(-y / \tau_{3}\right), \\
& M_{4} / M_{\max }=\frac{1}{1+B_{4} \exp \left(-y / \tau_{4}\right)} .
\end{aligned}
$$

\footnotetext{
${ }^{4}$ For the computation of the Mesolithic site densities (fig. 1), we have used an area $200 \mathrm{~km}$ wider than the rectangular region in fig. 1 in ref. [3] (100 km wider per side) in order to obtain a better statistics, as well as to include the effect of neighboring sites.
} 


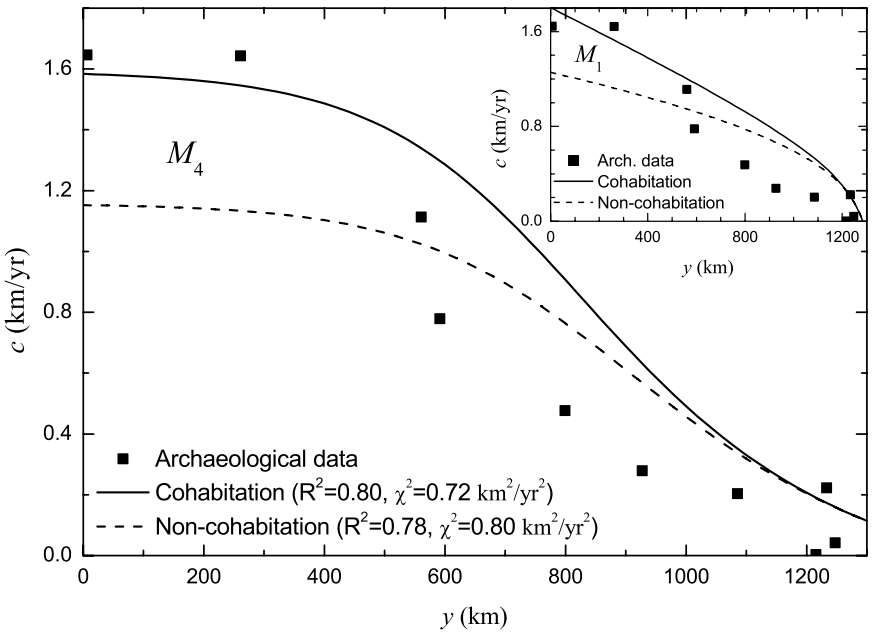

Fig. 2: Predicted speeds for the slowdown of the Neolithic in Europe for a non-cohabitation model (dashed line), eq. (5), and a cohabitation model (solid line), eq. (11) when fitting the Mesolithic population density to an S-shaped curve $\left(M_{4}\right)$. Symbols correspond to archaeological results for the front speed. Inset: results obtained when fitting the Mesolithic population density to a line $\left(M_{1}\right) \quad\left(\chi_{\text {Cohab }}^{2}=0.82 \mathrm{~km}^{2} / \mathrm{y}^{2}\right.$, $\left.R_{\text {Cohab }}^{2}=0.77, \chi_{\text {Non- } \text { cohab }}^{2}=0.88 \mathrm{~km}^{2} / \mathrm{y}^{2}, R_{\text {Non- } \text { cohab }}^{2}=0.75\right)$.

Such functions were already proposed in ref. [3], but they were not fitted to data in this reference. In contrast, fig. 1 includes the data (triangles) as well as the values of $\chi^{2}$ (residual sum of squares) and Adj. $R^{2}$ (adjusted coefficient of determination, which takes into account the effect of the degrees of freedom due to the number of fitting parameters [22]).

Clearly, the S-shaped curve ( $M_{4}$ in eq. (12)) is the model that provides the best approximation to the archaeological data for the Mesolithic population density, with the lowest value of $\chi^{2}\left(\chi^{2}=0.22\right)$ and the highest value of the adjusted coefficient of determination (Adj. $R^{2}=0.82$ ).

Figure 2 shows the front speeds predicted by the noncohabitation speed (5) and the cohabitation one (11) when the spatial dependency of the Mesolithic population density $M(y)$ is described by $M_{4}$. In this figure we can see that both equations for the front speed, (5) and (11), predict that the speed of the Neolithic front decreases with increasing distance $y$, as expected because i) $\tilde{a}$ decreases as $M$ increases and ii) the correction due to the nonisotropic dispersal kernel (second term in eqs. (5) and (11)) is higher as $M$ approaches $M_{\max }$. However, although both models lead to a similar behavior at large distances, for most of the range the front speeds predicted by the cohabitation equation (11) are substantially faster, up to $37 \%$, than those from the non-cohabitation equation (5). Moreover, by comparing the calculated speeds in fig. 2 with the archaeological data (squares), one can see that the new cohabitation equation (11) (full line) provides better predictions for the front speed (specially at southern regions, i.e., lower values of $y$ ), and this conclusion is statistically confirmed for the whole range as it yields a lower value of $\chi^{2}$ (and higher values of $R^{2}$, see footnote ${ }^{5}$ ) than the non-cohabitation equation (5) (dashed line) $\left(\chi_{\text {Cohab }}^{2}=0.72 \mathrm{~km}^{2} / \mathrm{y}^{2}\right.$ vs. $\chi_{\text {Non-cohab }}^{2}=$ $0.80 \mathrm{~km}^{2} / \mathrm{y}^{2}$, and $R_{\text {Cohab }}^{2}=0.80$ vs. $\left.R_{\text {Non-cohab }}^{2}=0.78\right)$. Thus we conclude that the new cohabitation model is able to give a satisfactory explanation of the slowdown of the absolute speed, whereas the non-cohabitation model (dashed line in fig. 2) is only able to explain the relative speed $c / c_{\max }$, with $c_{\max }=2 \sqrt{a D}$ (see fig. 3 in ref. [3]).

Moreover, even though they provide poorer fits to the Mesolithic density, the other three curves $\left(M_{1}\right.$, $M_{2}$ and $\left.M_{3}\right)$ in fig. 1 do also yield better predictions for the cohabitation speed (11) than for the noncohabitation one (5), thus reinforcing the conclusion that the cohabitation model can provide a satisfactory explanation of the absolute value of the speed as a function of distance $\left(\chi_{\text {Cohab }}^{2}=0.82 \mathrm{~km}^{2} / \mathrm{y}^{2}\right.$ vs. $\chi_{\text {Non-cohab }}^{2}=$ $0.88 \mathrm{~km}^{2} / \mathrm{y}^{2}$, and $R_{\text {Cohab }}^{2}=0.77$ vs. $R_{\text {Non-cohab }}^{2}=0.75$ for $M_{1}$ (see inset in fig. 2); $\chi_{\text {Cohab }}^{2}=0.83 \mathrm{~km}^{2} / \mathrm{y}^{2}$ vs. $\chi_{\text {Non-cohab }}^{2}=0.88 \mathrm{~km}^{2} / \mathrm{y}^{2}$, and $R_{\text {Cohab }}^{2}=0.77$ vs. $R_{\text {Non-cohab }}^{2}=0.75$ for $M_{2} ;$ and $\chi_{\text {Cohab }}^{2}=1.00 \mathrm{~km}^{2} / \mathrm{y}^{2}$ vs. $\chi_{\text {Non-cohab }}^{2}=1.03 \mathrm{~km}^{2} / \mathrm{y}^{2}$, and $R_{\text {Cohab }}^{2}=0.72$ vs. $R_{\text {Non-cohab }}^{2}=0.71$ for $M_{3}$ ).

Before closing this section, it is worth to note that the S-shaped function $M_{4}$ provides not only the best fit to the Mesolithic population density $\left(\chi^{2}\right.$ and Adj. $R^{2}$ values in fig. 1), but it is also the curve that yields better predictions for the front speed (compare the values of $\chi^{2}$ and $R^{2}$ in the main fig. 2 to those for $M_{1}, M_{2}$ and $M_{3}$ quoted in the previous paragraph).

Concluding remarks. - In this paper we have derived a new cohabitation reaction-diffusion equation for a population invading a range where there is a pre-existing, indigenous population which decreases the free space available for the newcomers, thereby diminishing their reproductive dynamics and opposing their dispersal capability. We have applied the new model to the slowdown of the Neolithic transition in Europe. The new cohabitation equation is more reasonable than non-cohabitation models when modelling human population dynamics, because it takes into account the fact that human populations migrate without leaving their children behind.

We have obtained an estimation of the variation of the Mesolithic relative population density from archaeological data and applied the results to the new cohabitation model as well as the previous non-cohabitation one. We have compared the results from both models for the slowdown of the Neolithic, and found that the new model leads to faster speeds, with substantial corrections (up to about $37 \%)$ relative to the previous, non-cohabitation model. Therefore, the cohabitation effect should be taken into

\footnotetext{
${ }^{5}$ Note that in fig. 2 we have not fitted the functions to the experimental points, so it is not necessary to adjust $R^{2}$ to the degrees of freedom. Thus $R^{2}$ is used in fig. 2, whereas [Adj. $R^{2}$ ] is used in fig. 1 .
} 
account when analyzing the front dynamics of interacting human populations in general, not only for the Neolithic transition in Europe. Moreover, we have compared both the cohabitation and the non-cohabitation models with the absolute speeds obtained from archaeological data (as opposed to the relative speeds, already analyzed in ref. [3]). This has lead us to the interesting conclusion that the new, cohabitation model (eq. (11)) provides a satisfactory explanation for the absolute speeds obtained from archaeological data, whereas the previous, noncohabitation model (eq. (5) and ref. [3]) does not.

$$
* * *
$$

Funded by the Ministry of Science (grants SimulPastConsolider-CSD-2010-00034 and FIS-2009-13050) and the Generalitat de Catalunya (Grup Consolidat 2009-SGR374). NI was supported by the MEC under the FPU program.

\section{REFERENCES}

[1] Fort J. and Pujol T., Rep. Prog. Phys., 71 (2008) 086001.

[2] Steele J., Hum. Biol., 81 (2009) 121.

[3] Isern N. and Fort J., New J. Phys., 12 (2010) 123002.

[4] Fort J. and Méndez V., Phys. Rev. Lett., 82 (1999) 867.

[5] Patterson M. A., Sarson G. R., Sarson H. C. and Shukurov A., J. Archaeol. Sci., 37 (2010 ) 2929.

[6] Fedotov S., Moss D. and Campos D., Phys. Rev. E, 78 (2008) 026107.

[7] Price T. D., The Widening Harvest. The Neolithic Transition in Europe: Looking Back. Looking Forward, edited by Ammerman A. J. and Biagi P. (Archaeological Institute of America, Boston, Mass.) 2003, p. 280.
[8] Coward F., Shennan S., Colledge S., Conolly J. and Collard M., J. Archaeol. Sci., 35 (2008) 42.

[9] Ackland G. J., Signitzer M., Stratfor K. and Cohen M. H., Proc. Natl. Acad. Sci. U.S.A., 104 (2007) 8714.

[10] FIX A. G., Migration and Colonization in Human Microevolution (Cambridge University Press, Cambridge, UK) 1999, p. 48.

[11] See fig. 4 in MacDonald D. H. and Hewlett B. S., Curr. Anthropol., 40 (1999) 501.

[12] Fort J., Pujol T. and Vander Linden M., Modelling the Neolithic transition in the Near East and Europe, to be published in Am. Antiq. (2012).

[13] Fort J., Pérez-Losada J. and Isern N., Phys. Rev. E, 76 (2007) 031913.

[14] Isern N., Fort J. and PÉrez-Losada J., J. Stat. Mech: Theor. Exp. (2008) P10012.

[15] Méndez V., Fort J. and Farjas J., Phys. Rev. E, 60 (1999) 5231.

[16] Pinhasi R., Fort J. and Ammerman A. J., PLoS Biol., 3 (2005) 2220.

[17] Fort J., Jana D. and Humet J., Phys. Rev. E, 70 (2004) 031913. See note [24] in this reference for estimation of the generation time $T=32$ years.

[18] Zimmermann A., Hilpert J. and Wendt K. P., Hum. Biol., 81 (2009) 357.

[19] Bocquet-Appel J.-P., Demars P.-Y., Noiret L. and Dobrowsky D., J. Archaeol. Sci., 32 (2005) 1656.

[20] Vermeersch P. M. and Boon J., Radiocarbon Paelolithic Europe Database. INQUA-Commission on Palaeoecology and Human Evolution, http://ees. kuleuven.be/geography/projects/14c-palaeolithic.

[21] The Mesolithic database used in the present paper is available in Excel format at http://copernic. udg. edu/QuimFort/fort.htm.

[22] Rawlings J. O., Pantula S. G. and Dickey D. A., Applied Regression Analysis : A Research Tool, 2nd edition (Springer, New York) 1998. 